\title{
Verrucous Carcinoma of Vulva: An Unusual Case Report
}

\author{
Patel $\mathbf{J}^{1}$, Masand $\mathrm{D}^{2}$ \\ ${ }^{1}$ Dr Jaya Patel, Post Graduate student, ${ }^{2}$ Dr Deepa Masand, Associate Professor. Both are affiliated with Department of \\ Obstetrics \& Genecology, National Institute of Medical Sciences, Shobhanagar, Jaipur (Rajasthan), India
}

Address for correspondence: Dr Jaya Patel, Email: sunshinejaya@gmail.com

\begin{abstract}
Introduction: Verrucous carcinoma of the vulva is a variant of squamous cell carcinoma and is a rare type of vulvar cancer, consisting 1-2 \% of vulvar cancer overall .It is locally invasive and rarely metastasize. The aetiology is not known. Case report: A 60 year old postmenopausal, multipara Indian woman presented in July 2012 with anexophytic irregular growth that was locally destructive associated with vulvar itching, pain and change in skin colour in the OPD of Obstetrics and Gynaecology department of NIMS Medical College, Jaipur (Rajasthan), India. She was a chronic smoker. Local examination shows 5-6 cm raised verrucous growth on the lower part of right labia majora with $1 \mathrm{~cm}$ ulcer at tip. Complete evaluation revealed verrucous carcinoma of vulva FIGO stage IB with no lymph node involvement. The patient was treated with an extensive excision of the damage and radiotherapy was advised.
\end{abstract}

Key words: Verrucous, Carcinoma, Vulva, HPV 6, HPV 8

\section{Introduction}

Verrucous carcinomas (VC) of the vulva represent a distinct entity. It is characterized with slow growing, rarely metastasizing to lymph nodes [1] and is presented as an exophytic appearing growth that can be locally destructive. The incidence of this type of malignancy is about $1-2 \%$ of all gynecological cancers. Verrucous carcinoma has also been found in the oropharynx, perianal, cervix, vagina, penis, scrotum, bladder and anorectal regions $[2,3]$.

\section{Case report}

A 60 year old postmenopausal, multipara Indian woman presented with an exophytic appearing irregular growth that was locally destructive associated with vulvar itching, pain and changing colour in the skin in the OPD of Obstetrics and Gynaecology department of NIMS Medical College, Jaipur. She was a chronic smoker but didn't take alcohol or any drug. Her family history did not reveal any malignancies in her and in first-degree relatives and her past medical history and surgical history was unremarkable. Patient was $78 \mathrm{~kg}$ obese lady with normal general physical examination and no lymphadenopathy. On vulvar examination colour changes were seen on both labia majora and adjacent skin of vulva. 5-6 cm raised verrucous lesion was seen on the lower part of right labia majora, ulcer of $1 \mathrm{~cm}$ diameter present on the tip of growth. On per speculum \& per vaginal examination cervix flushed with vagina, uterus was atrophic, no abnormality seen in cervix and vagina. All blood investigations, X-ray chest, MRI abdomen and pelvis along with biopsy of the growth sent for histopathological examination that shows well circumscribed "pushing" tumour invading the underlying stroma characterized by presence of club shaped figures of epithelium. The tumour was composed of cells with minimal nuclear atypia, hyper keratotic areas on the surface of tumour with little keratin formation inside the tumour and diffuse chronic inflammation of the stroma. No infiltration into the underline stroma was seen and HPV 6 confirmed by PCR. No sign of distant metastasis found. The complete evaluation revealed verrucous carcinoma of vulva FIGO stage $1 \mathrm{~B}$ with no lymph node involvement. The patient was treated with an extensive excision of the damage (vulvectomy). The histological findings confirmed the presence of verrucous carcinoma with tumour free margins. Following a decision, made by the gynaecological oncology team, it seemed appropriate for the patient to undergo radiotherapy. The patient is reviewed every six months thereafter with control imaging and physical and blood examination. The patient until now has not presented any other symptoms. 


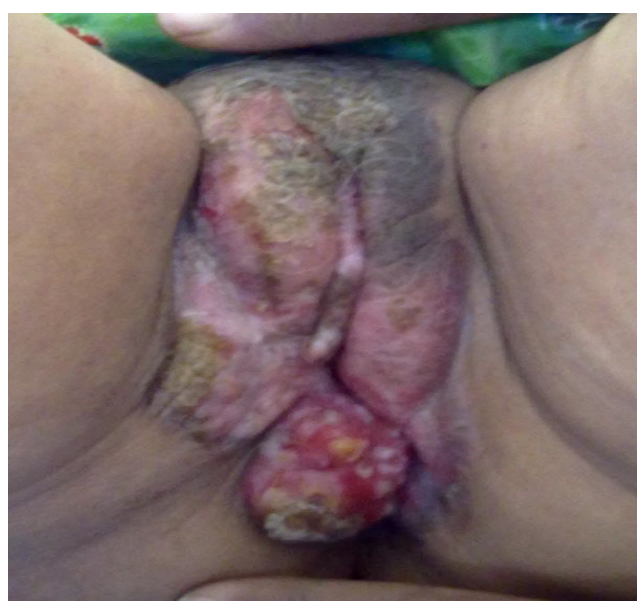

Fig 1: Carcinoma of Vulva

\section{Discussion}

Vulvar cancer is an uncommon lesion of the female genital tract. Vulvar carcinoma is encountered most frequently in postmenopausal women [4]. The cause of the lesion is not fully understood, but HPV-6 and HPV-11 were found to be associated in some studies, whereas others find it to have no association with HPV infection $[5,6]$. The different types of vulvar cancer are etiologically heterogeneous [7]. Verrucous carcinoma of the vulva is a variant of squamous cell carcinoma and is a rare type of vulvar cancer,constituting less than $1 \%$ of vulvar cancer overall. The aetiology of verrucous carcinoma is not known. However, there have been records showing the presence of HPV genome in the carcinoma tissue [8]. Risk factors for vulvar cancer include cigarette smoking, vulvar dystrophy (e.g.: lichen sclerosis), Vulvar or cervical intraepithelial neoplasia, Human papilloma virus (HPV) infection, Immunodeficiency syndromes, a prior history of cervical cancer, and northern European ancestry [9, 10]. Two independent pathways of vulvar carcinogenesis are felt to currently exist, the first related to mucosal HPV infection and the second related to chronic inflammatory (vulvar dystrophy) or autoimmune processes [11]. HPV has been shown to be responsible for 60 percent of vulvar cancers [12]. Specifically, HPV 16 and 33 are the predominant subtypes accounting for 55.5 percent of all HPV-related vulvar cancers [13].Verrucous carcinomas are locally invasive and rarely metastasize [14]. Verrucous carcinoma of the vulva may be difficult to treat. In fact, the treatment is still a matter of discussion. Surgery is considered the most effective treatment, but can be associated with local recurrences, especially when the tumor has been inadequately resected $[15,16]$. In addition, it is crucial to perform an extensive excision of the ill-defined disease, because of the potential invasion of deep adjacent structures. Verrucous carcinomas are resistant to radiotherapy and sometimes may undergo transformation to squamous cell carcinoma. In some cases, radiation may cause anaplastic transformation, even if this finding is not globally accepted yet [17-20].

\section{Conclusion}

So we conclude that although rare, vulvar carcinoma should be considered as a possibility when we see a case of an exophytic growth in female genital tract with risk factors for the disease.

\section{Funding: Nil}

\section{Conflict of interest: Nil}

Permission from IRB: Yes

\section{References}

1. Japaze H, Dinh TV, Woodruff JD, Verrucous carcinoma of the vulva: study of 24 cases. Obstetrics and Gynecology, 1982;60(4):462-466

2. Kraus FT, Perezmesa C. Verrucous carcinoma-Clinical and pathologic study of 105 cases involving oral cavity, larynx and genitalia. Cancer.1966;19(1):26-38

3. Shear M,Pindborg JJ. Verrucoushyperplasia of the oral mucosa. Cancer. 1980; 46(8):1855-1862

4. Siegel R, Ma J, Zou Z, Jemal A. Cancer Statistics2014. CA:Cancer J Clin. 2014;64(1):9-29

Available online at: www.ijmrr.in

154 | P a g e 
5. Kondi PA, Deligeorgi PH, Liapis A, et al.Human papillomavirus in verrucous carcinoma of vulva:an immune pathological study of three cases. Eur J GynaecolObstet 1998;19:319-320

6. GualcoM,BoninS,FogliaG,etal.Morphological and biologic studies on ten cases of verrucous carcinoma of the vulva supporting the theory of a discrete clinicopathologicentity.Int J Gynecol Cancer. 2003;13:317-324

7. Boutas I, Sofoudis C, Kalampokas E, Anastasopoulos C, etal. Verrucous Carcinoma of the Vulva: A Case Report. Case Reports in Obstetrics and Gynecology.2013;2013:3-5

8. Crowther ME, Shepherd JH, Fisher C.Verrucous carcinoma of the vulva containing human papillomavirus11. Case report. British Journal of Obstetrics and Gynaecology.1988;95(4):414-418

9. Ansink A. Vulvar squamous cell carcinoma. SeminDermatol 1996;15:51

10. Madsen BS, Jensen HL, van den Brule AJ. Risk factors for invasive squamous cell carcinoma of the vulva and vagina--population-based case-control study in Denmark. Int J Cancer 2008; 122:2827

11. DeKoning MN, Quint WG, Pirog EC. Prevalence of mucosal and cutaneous human papillomaviruses in different histologic subtypes of vulvar carcinoma. Mod Pathol 2008; 21:334

12. Monk BJ, Burger RA, Lin F, et al. Prognostic significance of human papillomavirus DNA in vulvar carcinoma. Obstetrics Gynecology 1995; 85:709
13. Insinga RP, Liaw KL, Johnson LG, Madeleine MM. A systematic review of the prevalence and attribution of human papillomavirus types among cervical, vaginal, and vulvar precancers and cancers in the United States. Cancer Epidemiol Biomarkers Prev 2008; 17:1611

14. S. Gallousis. Verrucous carcinoma: Report of three vulvar cases and review of the literature. Obstetrics and Gynecology.1972; 40(4):502-507

15. Chu QD, Vezeridis MP, Libbey NP, Wanebo HJ. Giant condylomaacuminatum (Buschke-Lowenstein tumour) of the anorectal and perianal regions: analysis of 42 cases. Diseases of the Colon and Rectum. 1994; 37(9):950-957

16. Bjørge $\mathrm{T}$, Dillner J, Anttila $\mathrm{T}$ et al. Prospective seroepidemiological study of role of human papillomavirus in non-cervical anogenital cancers. British Medical Journal.1997; 315(7109):646-649

17. Demian SDE, Bushkin FL, Echevarria RA. Perineural invasion and anaplastic transformation of verrucous carcinoma. Cancer.1973; 32(2):395-401

18. Proffitt SD, Spooner TR, Kosek JC. Origin of undifferentiated neoplasm from verrucous epidermal carcinoma of oral cavity following irradiation. Cancer. $1970 ; 26(2): 389-393$

19. Zaino RJ, Nucci M, Kurman RJ. Diseases of the vagina. Blaustein's Pathology of the Female Genital Tract.Springer, New York, USA, 2011; 317

20. Isaacs JH.Verrucous carcinoma of the female genital tract. Gynecologic Oncology.1976; 4(3):259-269

\section{How to cite this article?}

Patel J, Masand D. Verrucous Carcinoma of Vulva: An Unusual Case Report. Int J Med Res Rev 2014;2(2):153-155. doi:10.17511/ijmrr.2014.i02.15. 